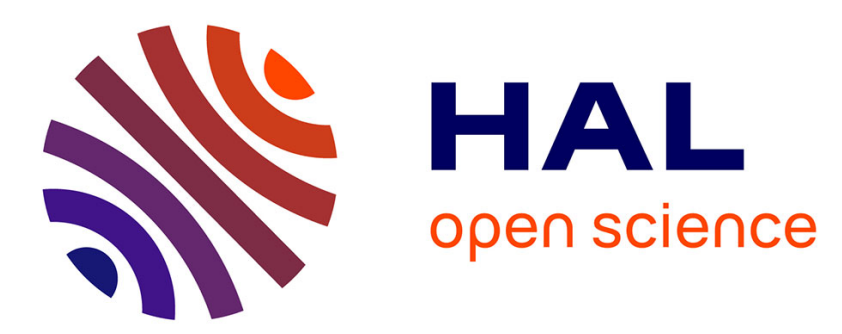

\title{
Revealing load hysteresis based on physical-mesomechanical deformation and fracture criteria
}

S. Yoshida, G.A. Gaffney, K. Yoshida

\section{To cite this version:}

S. Yoshida, G.A. Gaffney, K. Yoshida. Revealing load hysteresis based on physical-mesomechanical deformation and fracture criteria. Physical Mesomechanics, 2010, 13 (5), pp.337 - 343 . hal-01092763

\section{HAL Id: hal-01092763 \\ https://hal.science/hal-01092763}

Submitted on 9 Dec 2014

HAL is a multi-disciplinary open access archive for the deposit and dissemination of scientific research documents, whether they are published or not. The documents may come from teaching and research institutions in France or abroad, or from public or private research centers.
L'archive ouverte pluridisciplinaire HAL, est destinée au dépôt et à la diffusion de documents scientifiques de niveau recherche, publiés ou non, émanant des établissements d'enseignement et de recherche français ou étrangers, des laboratoires publics ou privés. 


\title{
Revealing load hysteresis based on physical-mesomechanical deformation and fracture criteria
}

\author{
S. Yoshida*, G.A. Gaffney and K. Yoshida \\ Southeastern Louisiana University, Hammond, LA 70402, USA
}

\begin{abstract}
Previously derived plastic deformation and fracture criteria are applied to an engineering application. Aluminum plate specimens are preloaded to various stress levels ranging from the elastic to plastic regime. After released from the preload, these specimens are reloaded at a stress level much lower than the yield stress. Electronic speckle pattern interferometry is used to observe fringe patterns that differentiate the level of preloading. Results of this study indicate that it is possible to reveal the load hysteresis through analysis of these fringe patterns.

Keywords: plastic deformation, load hysteresis, metal, electronic speckle pattern interferometry, physical-mesomechanical deformation criteria, diagnosis of deformation, interferometric fringe analysis
\end{abstract}

\section{Introduction}

Among a number of unique features, what makes physical mesomechanics [1] significantly distinctive over other theories of deformation and fracture, is its capability of describing different stages of deformation, i.e., the elastic, plastic and fracturing stage, on the same theoretical basis. This capability is based on the mesomechanical postulate: In the plastic regime deformation is still locally elastic and the dynamics can be described by considering the interactions among the local deformations. The physics underlying this postulate, known as the local (gauge) symmetry, states as follows: When a transformation representing a certain physical process is local (coordinate dependent), the associated Lagrangian must be invariant under the transformation so that the physics be the same before and after the transformation, and that to ensure the invariance a vector field called the gauge field must be introduced [2]. In the present context, this can be translated as follows: When an elastically deforming material enters the plastic regime, deformation starts to have a rotational mode; i.e., different parts of the material have their own degrees of rotation. This is one of the fundamental postulates of physical mesomechanics [1]. Consequently, the antisymmetric component of distortion tensor, known as the tensor of rigid body rotation in the elastic theories, represents deformation because different parts of the material experience different rotations.

\footnotetext{
* Corresponding author

Prof. Sanichiro Yoshida, e-mail: syoshida@selu.edu
}

In other words, the distortion tensor becomes coordinate dependent, and in order to preserve the gauge invariance a new vector field arises and that prescribes the dynamics of plastic deformation [3]. Similar concepts can be found in other fields of physics. A good example is that when we allow local symmetry in the phase transformation of charge particles, a vector field arises so that the corresponding physics (quantum mechanics) can be invariant [2]. The dynamics prescribed by this vector field is nothing but electrodynamics, and the vector field is the electromagnetic field. In fact, the field equations resulting from the physical-mesomechanical gauge symmetry are formulaically analogous to the well-known Maxwell equations of electrodynamics [3-5].

Previously, we discussed the physical-mesomechanical field equations in various occasions and contexts. In [6], we noted that one of the field equations represents an equation of motion describing the dynamics in which an unit volume in a plastically deforming material reacts to the external force through transverse restoring force and longitudinal damping force. When combined with other field equations, it yields a wave equation whose general solution is a decaying transverse wave of the displacement field. Here the oscillatory nature of the displacement field is caused by the transverse restoring force (restoring torque) and the decaying nature is due to the longitudinal damping force. The critical distinction from the elastic regime is that in the plastic regime the longitudinal force is not proportional to the stretch but to the local velocity; consequently, the work done by the external force is not stored in the field as the 
elastic energy but dissipates, making the associated deformation unrecoverable. These ideas have led to the plastic deformation and fracture criteria of initially isotropic and uniform materials [7]. When a material starts to exert restoring torque it is the beginning of plastic regime; at least part of the longitudinal force becomes energy dissipative. Subsequently, the material loses the recovery torque and the longitudinal damping force dominates; this is the prefracturing stage. Complete fracture can be interpreted as the situation where the material loses its mechanism of exerting the recoverable and damping force completely.

These ideas are supported by experimental observations. In [8], a plastic deformation wave is observed in an aluminum alloy specimen under a tensile load. It is demonstrated that the wave decays and when it completely decays the specimen fractures. In [9], a near-crack tip plastic zone is identified as the region where the plastic deformation criterion is satisfied, which subsequently develops to the prefracturing stage (the critical fracture criterion) and, within the following tenth of seconds, develops to a complete fracture. In these experiments, the displacement field was observed on a real-time basis with the use of an optical interferometric technique known as the electronic speckle pattern interferometry [10].

These experiments not only support the physical-mesomechanical plastic deformation and fracture criteria but also indicate the possibility of engineering applications of them. Apparently, the plastic deformation and fracture criteria applied in [9] to identify a near crack-tip plastic zone can be utilized as a method of fracture prediction. In this paper, we propose another application of the plastic deformation criterion using the same type of electronic speckle pattern interferometry as [9]. We demonstrate that by applying a low-level test tensile-load to previously loaded specimens and examining the resultant fringe patterns, it is possible to reveal the loading hysteresis. Based on the transition in the material response to the external force mentioned above, i.e., the longitudinal recovery force in the elastic regime, the combination of recovery torque and longitudinal damping force in the plastic regime, and the loss of recovery mechanism in the fracture stage, the fringe patterns are examined. Below after reviewing the physical-mesomechanical description of deformation dynamics with focus on the transition of material responses, we demonstrate this idea using a commercially available aluminum plates as sample specimens.

\section{Formulations}

\subsection{Physical mesomechanical field equations and dynamics}

The physical-mesomechanical field equations can be put in the following form $[3,6]$ :

$$
\nabla \cdot \mathbf{v}=\frac{\rho}{\varepsilon},
$$

$$
\begin{aligned}
& \nabla \times \mathbf{v}=\frac{\partial \boldsymbol{\omega}}{\partial t}, \\
& \nabla \times \boldsymbol{\omega}=-\varepsilon \mu \frac{\partial \mathbf{v}}{\partial t}-\mathbf{J},
\end{aligned}
$$

where $\mathbf{v}$ is the velocity of the deforming material at $(x, y, z)$, $\varepsilon$ is the density of the medium, $\rho / \varepsilon$ and $\mathbf{J}$ are, respectively, the time and spatial component of the charge of symmetry, $\boldsymbol{\omega}$ is the angle of the local volume element from its rotational equilibrium, and $1 / \mu$ is the shear modulus. Application of divergence to Eq. (3) with the use of Eq. (1) leads to an equation of continuity:

$$
\frac{\partial \rho}{\partial t}=-\frac{1}{\mu}(\nabla \cdot \mathbf{J}) \text {. }
$$

By understanding $\mathbf{J} / \mu$ as the momentum loss associated with a flow of strain concentration (see below), this equation of continuity can be interpreted as follows: the only way for the charge to change its density over time is the net momentum loss at the boundaries of the flow. Equation (4) allows us to express the current in the following form

$$
\frac{\mathbf{J}}{\mu}=\rho \mathbf{W}_{\mathrm{d}}=\varepsilon(\nabla \cdot \mathbf{v}) \mathbf{W}_{\mathrm{d}},
$$

where $\mathbf{W}_{\mathrm{d}}$ is the drift velocity of the charge density $\rho$ and Equation (1) was used.

According to fluid dynamics, the change in the momentum $\mathbf{p}$ of a unit volume of a flowing medium subject to an external force in the direction of its velocity per unit time is given as

$$
\frac{\mathrm{d} \mathbf{p}}{\mathrm{d} t}=\varepsilon(\nabla \cdot \mathbf{v}) \mathbf{v} .
$$

The resemblance between the right-hand sides of Eqs. (5) and (6) indicates that $\mathbf{J} / \mu$ is some sort of force. Indeed, it is possible to interpret it as the longitudinal force acting on a unit volume of a deforming material in the direction of the local velocity [6]. It is also possible to show that in the elastic limit, $\mathbf{J} / \mu$ represents the elastic force proportional to the displacement of the unit volume from the equilibrium state [11]. These arguments allow us to put $\mathbf{J} / \mu$ in the following form:

$$
\frac{\mathbf{J}}{\mu}=\sigma \mathbf{v} \text {. }
$$

The quantity $\sigma$ in Eq. (7) corresponds to conductivity in electrodynamics [5].

Rearrangement of Eq. (3) leads to

$$
\varepsilon \frac{\partial \mathbf{v}}{\partial t}=-\frac{1}{\mu}(\nabla \times \boldsymbol{\omega})-\frac{\mathbf{J}}{\mu} .
$$

Equation (8) can be interpreted as the equation of motion governing the unit volume, where the left-hand side represents the change in momentum and the right-hand represents the external force. A recent study [6] reveals that the first term on the right-hand side represents the recoverability mechanism in plasticity from the shear force associated with material rotation, and the second term the energy dissipating force associated with the loss in momentum [6]. Since 
Equation (8) holds when the left-hand side is zero leading to $\nabla \times \boldsymbol{\omega}=-\mathbf{J}$, it is possible to say that the current flows along the boundary of rotations. Note that $\varepsilon$ and $\mu$ are material constants: from this viewpoint, Equation (8) can be viewed as a constitutive equation as well.

\subsection{Plastic deformation and fracture criteria}

From the gauge theoretical viewpoint, as mentioned above, plastic deformation is characterized as the situation in which the distortion tensor is coordinate dependent. From this, we can assume that when the left-hand side of Eq. (3) is non-zero, the condition for plastic deformation is satisfied. In fact it is possible to show that when we set the lefthand side to zero, Equation (3) reduces to the elastic wave equation [11]. Thus the plastic deformation criterion can be given as below [7]:

$$
\nabla \times \boldsymbol{\omega} \neq 0 \text {. }
$$

Condition (9) means that if different regions of a material rotate differently, the deformation is plastic, in consistence with physical mesomechanical postulate [1]. On the other hand, if the entire region of the material experiences a common rotation, the deformation is elastic.

The prefracturing stage can be interpreted as the final stage of plastic deformation where the material loses its recoverability mechanism via the first term of the right-hand side of Eq. (8). The difference from elastic deformation, where $\nabla \times \boldsymbol{\omega}$ is zero, is that the current $\mathbf{J}$ is nonzero, causing the energy dissipation. Thus the critical fracture criterion [7] can be given as

$$
\begin{aligned}
& \nabla \times \boldsymbol{\omega}=0, \\
& \frac{\mathbf{J}}{\mu}=\varepsilon(\nabla \cdot \mathbf{v}) W_{\mathrm{d}} \neq 0 .
\end{aligned}
$$

These equations indicate that by monitoring $\boldsymbol{\omega}$ and $\nabla \cdot \mathbf{v}$, it is possible to diagnose the plastic deformation and fracturing stages of the object. Complete fracture can be char-

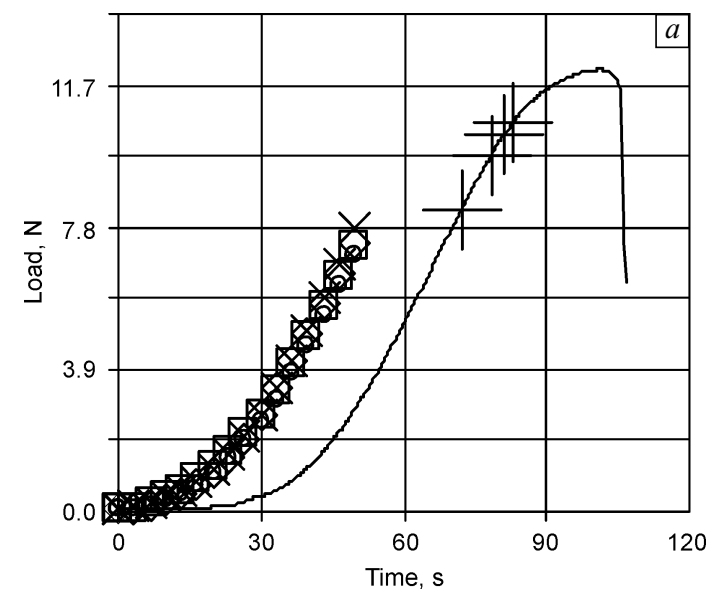

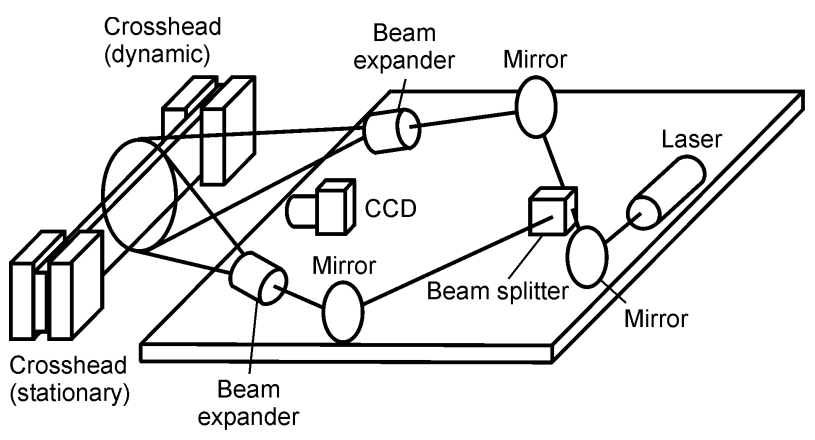

Fig. 1. Experimental arrangement

acterized as the stage where even $\mathbf{J}$ becomes zero, i.e., the material loses the resisting mechanism completely. Below, we demonstrate an idea of monitoring these quantities via interferometric fringe patterns that represent the displacement field of a deforming object.

\section{Experiment}

\subsection{Displacement measurement with electronic speckle pattern interferometry}

Figure 1 illustrates the experimental arrangement for this study. We set up a dual beam electronic speckle pattern interferometry sensitive to in-plane displacement [10] in front of an aluminum specimen attached to a tensile machine, using a continuous-wave, helium-neon laser oscillating at $632.8 \mathrm{~nm}$ as the light source. We split the laser beam into two paths on a horizontal plane, configuring the resultant two beams as two interferometric arms to illuminate the surface of the specimen at the same angle of incidence and created the sensitivity in the direction of the tensile axis. The CCD camera captured the image of the specimen at a frame rate of 30 frame/s. We sent captured images into computer memory and subtracted each image from the

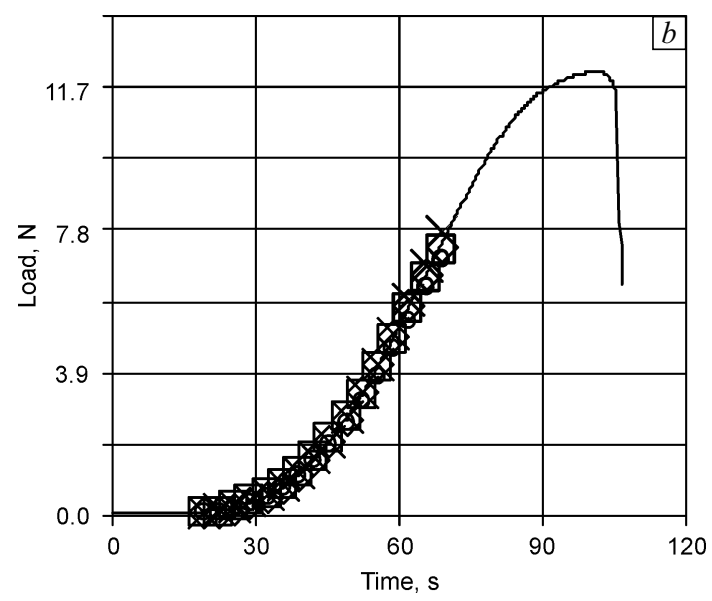

Fig. 2. Loading characteristics of full load and reloads. The reload characteristics are shifted on the right figure so that rising slopes can be compared with the full loading characteristics. Specimens $A(\diamond), B(\square), C(\mathrm{O}), D(\times) ;-$ full load, $\mathbf{-}$ - preload $A, B, C, D$ 


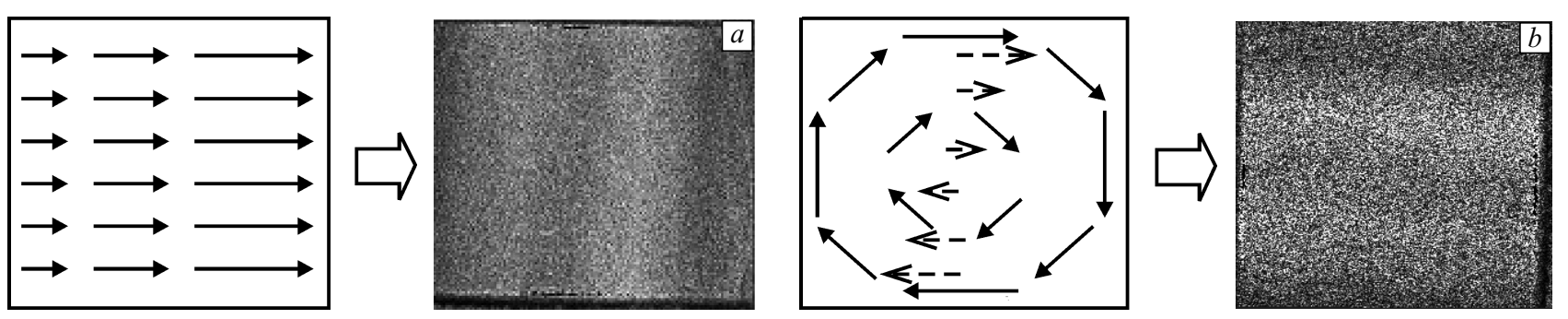

Fig. 3. Fringe patterns representing uniform stretch $(a)$ and pure rotation $(b)$. The dashed lines in the right picture denote the horizontal components of the displacement under pure rotation, which a horizontally sensitive interferometer is sensitive to

image captured at a later frame, typically 10-20 frames later. We set the interval between the images of subtraction such that the result of subtraction would provide an appropriate number of interferometric fringes. Thus the interval depended on the tensile speed. We monitored the fringes on a real-time basis using our home-made image processing software.

\subsection{Preload and reload test}

The specimen used in this study was a $100 \mathrm{~mm}$ long, $20 \mathrm{~mm}$ wide and $0.5 \mathrm{~mm}$ thick rectangular aluminum plate with shallow notches at the center of the $100 \mathrm{~mm}$ sides. We prepared a number of specimens with these dimensions and conducted the following experiments. To obtain a base-line data, we first applied a tensile load to a specimen at a constant head speed of $20 \mu \mathrm{m} / \mathrm{s}$ until it broke. The solid line labeled "full load" in Fig. 2 shows the loading characteristics of this experiment. Next we prepared four groups of specimens by preloading them at the following four different stress levels; $8.3 \mathrm{~N}$ (specimen $A), 9.8 \mathrm{~N}$ (specimen $B$ ), $10.4 \mathrm{~N}$ (specimen $C$ ), and $10.7 \mathrm{~N}$ (specimen $D$ ). Finally, we reloaded all these specimens up to a stress level of $7.9 \mathrm{~N}$ five times, forming fringe patterns with the electronic speckle pattern interferometry shown in Fig. 1. The four preload levels are indicated in Fig. 2 along with the reloading characteristics of the respective specimens. Note that the maximum reload level of $7.9 \mathrm{~N}$ is within the linear range of the loading characteristics when the specimen was pulled until fracture (the full loading characteristics). In the right plot of Fig. 2, we shift the reloading characteristics on the time scale so that the initial rise of the reload overlaps the full loading curve. Notice that all the preloaded specimens show reloading characteristics perfectly overlapping each other and the full loading characteristics. This indicates that it is impossible to reveal the loading hysteresis by examining the reloading characteristics.

\section{Results and discussions}

To facilitate the discussion regarding the fringe patterns in connection with the displacement field of the examined specimen, let us first consider general pattern of fringes. Refer to Fig. 3 and consider the fringes observed with an electronic speckle pattern interferometry setup sensitive to horizontal displacement. When the specimen experiences horizontal stretch to the right, for example, the right end of the specimen displaces the most largely, the left end displaces the least and the rest parts displace in proportion to the distance from the left end. Consequently, the fringe pattern consists of vertical straight lines. If the deformation is uniform, the vertical lines are equally spaced. On the other hand, when the specimen experiences pure rotation, the fringe pattern observed in the same electronic speckle pattern interferometry setup consists of horizontal straight lines. If the deformation is a combination of these, which is normally the case, the resultant fringes are mixture of these patterns.

When reloaded, the preloaded specimens generally show the following trends. As the applied load was increased from $0 \mathrm{kgf}$ to $7.9 \mathrm{~N}$, the fringe patterns show changes that can be classified into five stages. Figure 4 shows representative fringe patterns observed in each stage. The actual stress level at which the fringe pattern changes from one stage to the next depends on the preloading condition and the number of reloading, as indicated by Figs. 5-7.

These transitions in the stage of fringe patterns can be explained in terms of the recovery mechanism in plasticity in conjunction with the fracture criterion represented by Eqs. (10) and (11). As the deformation develops, the material loses the recoverability mechanism (the plastic recovery force $\nabla \times \boldsymbol{\omega} / \mu$ represented by the first term of Eq. (8)) and instead, the energy dissipation mechanism (the current $\mathbf{J} / \mu$ represented by the second term) dominates. In a twodimensional picture on an $x y$-plane, the plastic recovery

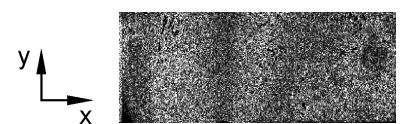

Stage 1

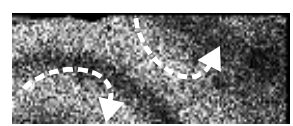

Stage 2

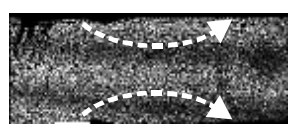

Stage 3

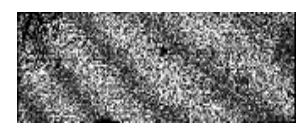

Stage 4

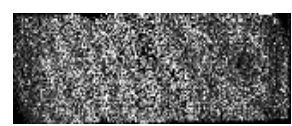

Stage 5

Fig. 4. Representative fringe-pattern observed in each stage. The arrows indicate rotation of the material 


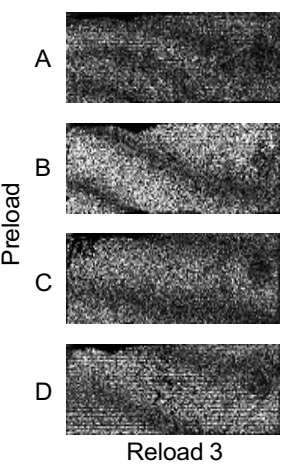

Reload 3
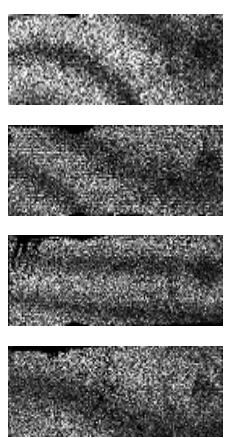

Reload 4
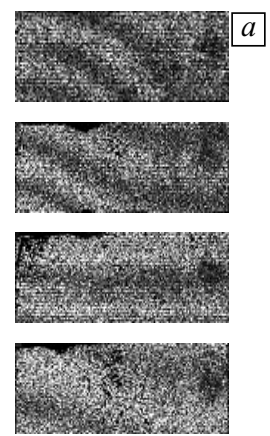

Reload 5
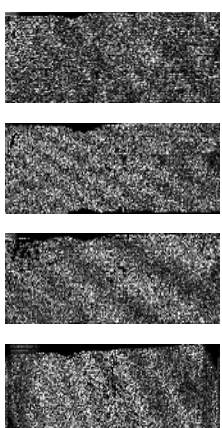

Reload 3
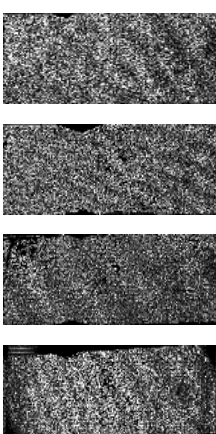

Reload 4
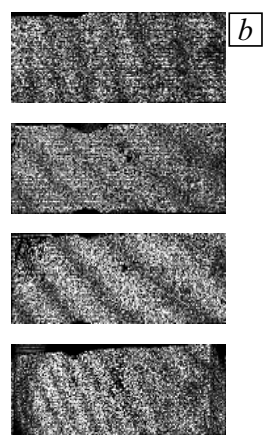

Reload 5

Fig. 5. Fringe patterns observed with reload level of $0.98(a)$ and $4.9 \mathrm{~N}(b)$

force and the current can be expressed in terms of the inplane displacement and rotation around an axis normal to the plane. Under the present experimental condition, since the interferometer has the sensitivity in the horizontal $x$ component of the in-plane displacement on the $x y$-plane, the plastic recovery force and the current are detected in the fringe pattern only in the spatial variation of the horizontal displacement as shown below:

$$
\begin{aligned}
& \frac{1}{\mu}(\nabla \times \boldsymbol{\omega})=\frac{1}{\mu}\left(\frac{\partial \omega_{z}}{\partial y} \hat{\mathbf{x}}-\frac{\partial \omega_{z}}{\partial x} \hat{\mathbf{y}}\right)= \\
& =\frac{1}{\mu}\left[\left(\frac{\partial^{2} v}{\partial x \partial y}-\frac{\partial^{2} u}{\partial^{2} y}\right) \hat{\mathbf{x}}-\left(\frac{\partial^{2} v}{\partial^{2} x}-\frac{\partial^{2} u}{\partial x \partial y}\right) \hat{\mathbf{y}}\right]= \\
& =\frac{1}{\mu}\left(-\frac{\partial^{2} u}{\partial^{2} y} \hat{\mathbf{x}}+\frac{\partial^{2} u}{\partial x \partial y} \hat{\mathbf{y}}\right),
\end{aligned}
$$

$$
\frac{\mathbf{J}}{\mu}=\varepsilon(\nabla \cdot \mathbf{v}) \mathbf{W}_{\mathrm{d}}=\varepsilon\left(\frac{\partial u}{\partial x}+\frac{\partial v}{\partial y}\right) \mathbf{W}_{\mathrm{d}} .
$$

Here $\omega_{z}$ is the $z$-component of the rotation, $u$ and $v$ are the $x$ - and $y$-components of the displacement, and $\hat{\mathbf{x}}$ and $\hat{\mathbf{y}}$ are the unit vectors. Note that in Eqs. (12) and (13) the $y$-and $z$-components of $\boldsymbol{\omega}$ and the terms containing $v$ are dropped because the interferometer does not have sensitivity to these components. With these expressions, the fringe pattern of each stage can be explained as below.

In stage 1 , the fringe patterns are approximately vertically parallel, indicating that the deformation is basically elastic. If the deformation is purely elastic, the fringes representing displacement $u$ observed in the horizontally sensitive interferometer ought to be equidistant, vertical straight lines. As the left-most image in Fig. 4 shows, the fringe

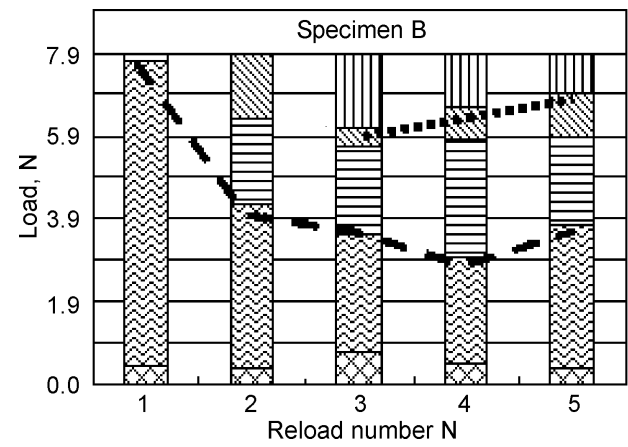

Stage

ए 1

ㅇ 2

日 3

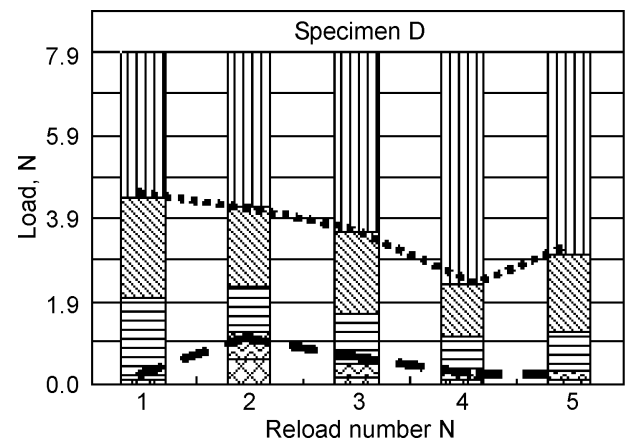

용 4

지 5

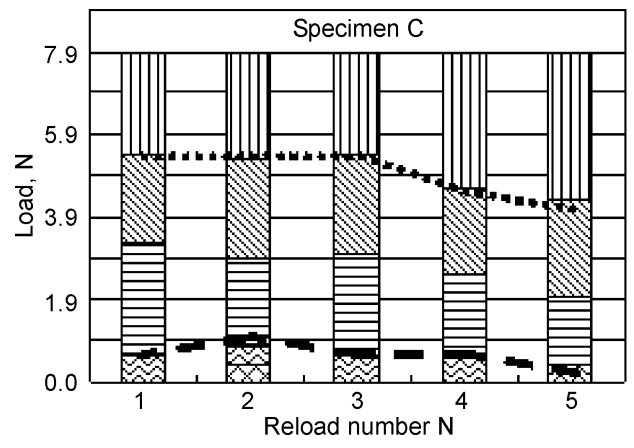

Fig. 6. Change in fringe patterns as reloading number increases 

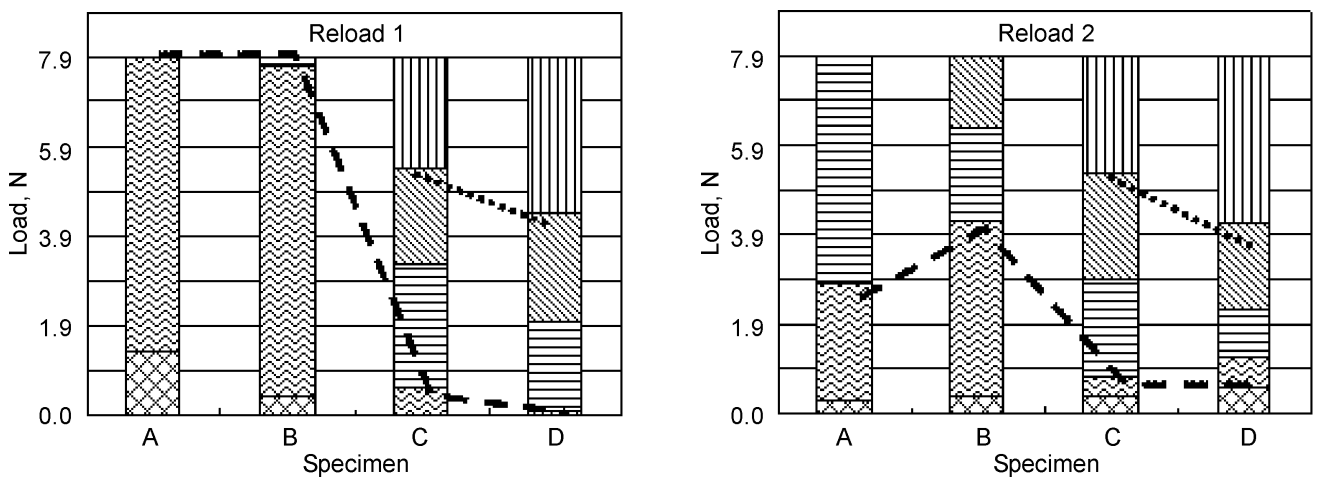

\section{Stage}

प 1

요 2
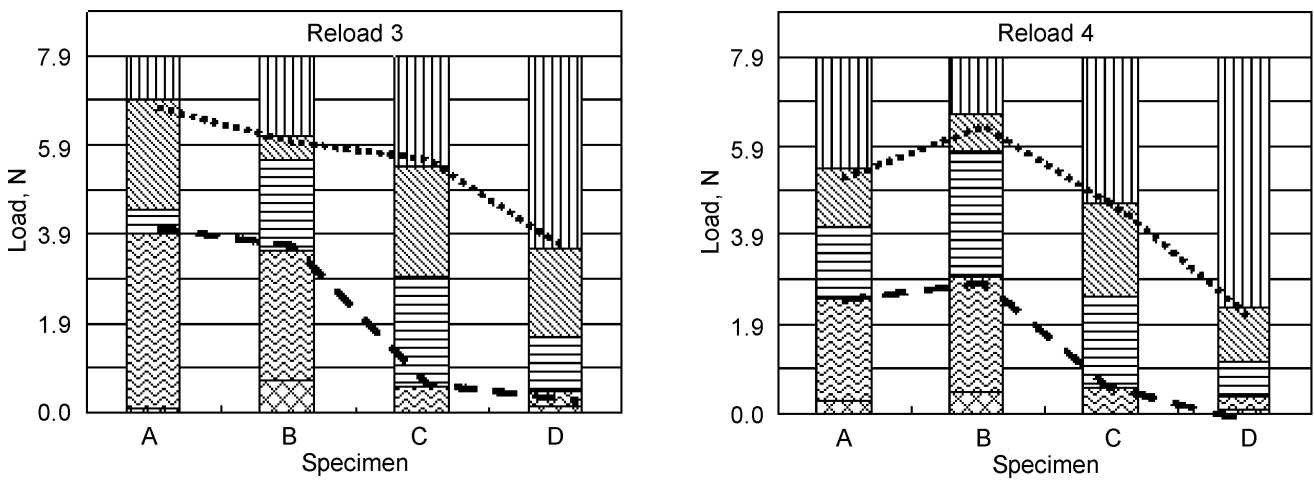

Fig. 7. Change in fringe patterns as preload level increases for each reload

patterns seen in stage 1 are normally not completely parallel indicating that the deformation in this stage is not purely elastic. This is consistent with the fact that the loading curve is not completely linear in the initial rising part.

In stage 2 , the fringe patterns consist of horizontal wavy lines, indicating that $(\nabla \times \boldsymbol{\omega})_{x}=\partial \omega_{z} / \partial y \neq 0$ and $(\nabla \times$ $\boldsymbol{x} \boldsymbol{\omega})_{y}=-\partial \omega_{z} / \partial x \neq 0$. Referring to Eq. (12), this represents the situation where neither of the $x$ - and $y$-components of the plastic recovery force is zero. Thus it is interpreted that in this stage, the material has the recovery mechanism both in parallel and perpendicular to the tensile load.

In stage 3 , the fringe patterns consist of horizontal straight lines. This pattern can be interpreted as $(\nabla \times \boldsymbol{\omega})_{x}=$ $=\partial \omega_{z} / \partial y \neq 0$ and $(\nabla \times \omega)_{y}=-\partial \omega_{z} / \partial x=0$, i.e., the material has lost the recovery force in the direction perpendicular to the tensile axis but still possesses its component parallel to the tensile load.

In stage 4, the horizontal lines observed in stage 3 begin to rise. As the load increases, they keep rising until they are mostly vertical in stage 5 . This transition can be interpreted as the situation where the material are losing the recovery force parallel to the tensile load as well $(\nabla \times \omega)_{x}=$ $=\partial \omega_{z} / \partial y=0$, and the energy dissipation mechanism represented by Eq. (13) becomes dominant.

In stage 5 , the fringes stop rotating and become mostly vertical, indicating that the recovery force associated with $\nabla \times \boldsymbol{\omega}$ is not effective $\left((\nabla \times \boldsymbol{\omega})_{x}=\partial \omega_{z} / \partial y=0\right.$ and $(\nabla \times$ $\left.\boldsymbol{x} \boldsymbol{\omega})_{y}=-\partial \omega_{z} / \partial x=0\right)$. The fringe pattern can be interpreted as representing the $(\partial u / \partial x+\partial v / \partial y)$ term in Eq. (13).

While specimens $A-D$ commonly show the transitions from stage 1 through stage 5 , the load level at which the fringe pattern changes from one stage to the next depends on the preload and reload conditions. Figure 5(a) shows typical fringe patterns observed in the third through fifth reloads in specimen $A-D$ at the reload level of $0.98 \mathrm{~N}$. Here the rows represent the preload conditions $(A-D$ from the top to bottom), and the columns represent the reload (thirdfifth reload from the left to right). The fringe patterns seen in the top two rows (in specimens $A$ and $B$ ) are typical wavy fringes observed in stage 2 . The fringes seen in the two right columns in the third row are straight fringes observed in stage 3 . The fringes seen in the bottom row are the rising fringes observed in stage 4 . Notice that in specimen $C$ (third row), the horizontal fringes are becoming more straight as the number of reload increases, indicating that the transition from stage 2 to stage 3 takes place as the reloading repeats. From this viewpoint, the leftmost pattern in the third row is an intermediate pattern between the "stage 2 wavy" and "stage 3 straight" fringes.

Similarly, Figure 5(b) shows typical fringes observed at the higher reload level of $4.9 \mathrm{~N}$. The row and column arrangements are the same as Fig. 5(a). The fringe patterns seen in this figure are basically the rising fringes in stage 4 . Notice that in all rows the fringes become more upright as going from the left to right. This indicates that as the re- 
loading repeats, the specimen experiences transition toward stage 5 .

Generally speaking, Figure 5 indicates that the higher the preloading level or the number of reloading, the specimen tends to experience the transitions from the initial to final stages at lower reload levels. Figures 6 and 7 present this trend more explicitly, where the former sorts out the data in terms of the preload conditions and the latter sorts out in terms of the number of reloading. The dashed line indicates the variation in the transition from stage 2 to stage 3 , i.e., the load level at which the material loses the ability to exert the recovery force in the direction perpendicular to the applied load. The dotted line indicates the variation in the transition from stage 3 to stage 4, i.e., the load level at which the material loses the recoverability in the direction parallel to the tensile axis as well. The following observations are found.

Observation 1. As the reloading is repeated, generally speaking, the transitions from one stage to the next take place at lower reload levels.

Observation 2. The above trend is more prominent in specimens $A$ and $B$ than specimens $C$ and $D$. In particular, the reload level that causes the transition from stage 2 to stage 3 reduces remarkably from the first to second reloading in specimen $A$ and $B$.

Observation 3. In all reloads, specimen $A$ and $B$ show similar trends and specimens $C$ and $D$ show similar trends, respectively. However, trends observed in the two groups are different from each other.

Observation 4 . Specimens $C$ and $B$ barely show stage 2 fringes, indicating that when this sample is preloaded to the level of $10.4 \mathrm{~N}$ or higher, the critical fracture criterion is almost satisfied so that the material barely exerts the recovery force from the beginning of reloads.

Observation 5. In the first reload, specimens $A$ and $B$ show stage 2 pattern till the end of reload $(7.9 \mathrm{~N})$. In the second reload and after, they show stage 3 pattern. This strongly indicates that the specimen $A$ and $B$ experience fatigue in the first reload, and that the effect caused by the mechanism of fatigue is similar to the effect caused by the tensile load.

Notice that whereas the above observations and the features observed in the fringe patterns enable us to diagnose the preload level, Figure 2 indicates that the specimens preloaded to different levels show the same reloading characteristics. This indicates that for the purpose of revealing loading hysteresis, it is necessary to analyze the spatial distribution of the displacement as seen in the fringe patterns. The loading characteristic, being representing the total displacement, is unable to reveal the loading hysteresis.

\section{Conclusion}

In summary, the dynamics of plastic deformation has been discussed based on the gauge theoretical formalism of physical mesomechanics. The fracture is formulated as the final stage of deformation where the material loses its mechanism of exerting resistive force, and thereby becomes totally energy dissipative. The constitutive equation of plasticity derived from the physical mesomechanical field equations has been discussed in connection with the transitional stage from plastic deformation to fracture. These concepts have been applied to an attempt of revealing the loading hysteresis of aluminum specimens. The specimens have been preloaded to four different stress levels, and after released from the preload, they are reloaded within the linear range of the loading characteristics. The change in the displacement field observed in the reloaded specimens has been analyzed with the use of an in-plane sensitive electronic speckle pattern interferometry. It has been found that through analysis of the observed fringe patterns, it is possible to differentiate the preload conditions one from another. Note that the reloading characteristics in the corresponding timeload diagram are found identical among different preload conditions, and therefore cannot be used to reveal the loading hysteresis. The observed features of the fringe patterns have been explained in accordance with the derived constitutive equation and fracture criterion.

\section{Acknowledgment}

The authors are grateful to Southeastern Louisiana University Alumni Association for financial support and T. Sasaki of Niigata University for various discussions.

\section{References}

[1] Physical Mesomechanics of Heterogeneous Media and ComputerAided Design of Materials, Ed. by V.E. Panin, Cambridge Interscience Publishing, Cambridge, 1998.

[2] I.J.R. Aitchison and A.J.G. Hey, Gauge Theories in Particle Physics, IOP Publishing, Bristol, 1989.

[3] V.E. Panin, Yu.V. Grinyaev, V.E. Egorushkin, I.L. Buchbinder and S.N. Kul'kov, Spectrum of excited states and the rotational mechanical field, Sov. Phys. J., 30, No. 1 (1987) 24.

[4] V.E. Egorushkin, Dynamics of plastic deformation: Waves of localized plastic deformation in solids, Rus. Phys., 35, No. 4 (1992) 316.

[5] S. Yoshida, Interpretation of mesomechanical behaviors of plastic deformation based on analogy to Maxwell electromagnetic theory, Phys. Mesomech., 4, No. 3 (2001) 29.

[6] S. Yoshida, Dynamics of plastic deformation based on restoring and energy dissipative mechanisms in plasticity, Phys. Mesomech., 11, No. 3-4 (2008) 137.

[7] S. Yoshida, Consideration on fracture of solid-state materials, Phys. Lett. A, 270 (2000) 320.

[8] S. Yoshida, B. Siahaan, M.H. Pardede, N. Sijabat, H. Simangunsong, T. Simbolon and A. Kusnowo, Observation of plastic deformation wave in a tensile-loaded aluminum-alloy, Phys. Lett. A, 251 (1999) 54.

[9] S. Yoshida, R.L. Rourks, T. Mita and K. Ichinose, Physical mesomechanical criteria of plastic deformation and fracture, Phys. Mesomech., 12, No. 5-6 (2009) 249.

[10] Speckle Metrology, Ed. by R.S. Sirohi, Marcel Dekker, New York, 1993.

[11] S. Yoshida, T. Sasaki and J.A. Gaffney, Dynamics of plastic deformation based on a field theory, Presented at Annual Conference \& Exposition on Experimental and Applied Mechanics, June 1-4, 2009, Albuquerque, New Mexico. 